\title{
CHARACTERIZATION OF POLYMERIC METAL-INSULATOR-SEMICONDUCTOR DIODES
}

\author{
S. Grecu , M. Bronner , A. Opitz* , and W. Brütting \\ Experimentalphysik IV, Universität Augsburg, 86135 Augsburg, Germany
}

\begin{abstract}
Metal-insulator-semiconductor (MIS) diodes are the two-terminal pendants of thin film transistors sharing the same basic layer structure. However, instead of the current-voltage characteristics one has to study the capacitance-frequency and capacitance-voltage behavior, which can give information about doping, mobile charges and trapping processes in these devices. We have investigated MIS structures based on poly(alkyl-thiophene) as semiconductor which were fabricated on glass substrates with polymeric insulator layers and compared their response to devices fabricated on $\mathrm{Si} / \mathrm{SiO}_{2}$ substrates. From capacitance-voltage measurements the acceptor dopant concentration is determined for different preparation conditions. Typically these measurements show hysteresis between forward and reverse bias sweeps. We have investigated this behavior as a function of external parameters like sweep speed and temperature and discuss their possible origin. The analysis of the frequency response using appropriate equivalent circuits allows the extraction of material parameters, like conductivity and charge carrier mobility, which are compared to data obtained on thin film transistor structures.
\end{abstract}

Key words: organic transistor, MIS diode, polymer insulator, polythiophene, 


\section{Introduction}

In recent years organic polymers became very attractive for building electronic devices as active matrix displays and all-polymer integrated circuits $[1,2]$. Features that make them so interesting are their low cost and solution processability, but at the same time there are a few drawbacks. In spite of the considerable amount of work done on these materials the problems still to be solved are lower mobility, compared to evaporated organic materials, stability and interface states which can affect considerably the electric properties of the devices [3]. The regioregular poly(3-alkylthiopenes) (P3HT), in particular poly(3-hexylthiophene), seem to be good candidates for organic electronics due to their relatively high field effect mobility and easy processability $[4,5]$.

In this paper, we present a comparison between two types of metal-insulatorsemiconductor (MIS) devices. One structure is based on $\mathrm{Si} / \mathrm{SiO}_{2}$ substrates, the other one uses indium-tin oxide (ITO) coated glass substrates covered with a solution processed polymer as gate insulator. By this way we can directly compare the effect of the organic-organic interface on the electrical properties of MIS diodes, which will also affect transistor performance. We have measured capacitance-frequency and capacitance-voltage curves in the dynamic regime and tried to correlate them with the measured output and transfer

\footnotetext{
* Corresponding author

Email address: andreas.opitz@physik.uni-augsburg.de (A. Opitz).
} 
characteristics of field effect transistors.

\section{Experimental}

\subsection{Preparation}

Two kinds of MIS diodes were used, with inorganic and organic gate dielectric. The schematic picture of the structures is given in Fig. 1(a). The inorganic type has been fabricated on highly p-doped silicon wafers with $50 \mathrm{~nm}$ of thermally grown silicon oxide and the organic type on indium-tin oxide (ITO) coated glass using an organic aromatic oligomeric resin capable of thermal or photo crosslinking in a butanol based formulation (from Merck). The organic dielectric was spin-coated from solution resulting in a film thickness of about $600 \mathrm{~nm}$ followed by thermal crosslinking (5 hours at $340 \mathrm{~K}$ ). The relative dielectric constant of this material is about 5 . In both cases smooth, homogenous P3HT films of about $150 \mathrm{~nm}$ thickness were spin-coated from $5 \%$ wt. solution in toluene, at 1500 revolutions per minute. There was a subsequent thermal treatment at $340 \mathrm{~K}$ for 10 hours. After this annealing step rectangular contacts with an area of $36 \mathrm{~mm}^{2}$ were produced by thermal evaporation of gold trough a shadow mask. P3HT (its structure is shown in Fig. 1(b)) was also obtained from Merck. Transistor structures were fabricated on $\mathrm{Si} / \mathrm{SiO}_{2}$ substrates in the bottom-gate, bottom-contact configuration with lithographically patterned source and drain electrodes from gold defining a channel width of $20 \mu \mathrm{m}$. In this case the P3HT layer has been prepared also by spin-coating from a $1 \%$ wt solution in toluene at $2000 \mathrm{rpm}$. The resulting film thickness was about $50 \mathrm{~nm}$. The solution processing of both, the polymeric insulator 
and P3HT, was performed in a nitrogen-filled glove-box system with partial pressures of oxygen and water below $1 \mathrm{ppm}$.

\subsection{Measurement details}

The capacitance-voltage $(C-V)$ and capacitance-frequency $(C-f)$ dependence of the devices was measured using a Solartron 1260 Impedance/Gain-phase Analyzer coupled with a Solartron 1296 Dielectric Interface in the frequency range from 1 to $10^{6} \mathrm{~Hz}$. All the measurements were carried out in a liquid nitrogen cooled cryostat (Cryovac) under vacuum or nitrogen atmosphere. In the case of $C$ - $f$ measurements there was a hold time of 5 seconds for the applied bias and 7 points/decade were measured with 1 second delay, for the $C-V$ measurements the hold time was 5 seconds and the ramp $0.5 \mathrm{~V} / \mathrm{s}$.

\section{Results and discussion}

\subsection{MIS diode with $\mathrm{SiO}_{2}$ insulator}

The hybrid structure of silicon dioxide and the polymer semiconductor was used for determining the doping concentration from $C-V$ curves and the mobility from $C$ - $f$ curves by two different approaches [6].

Fig. 2(a) shows two $C$ - $V$ curves measured at 270 and $330 \mathrm{~K}$. The curves show the typical step-like transition from accumulation at negative bias (with the capacitance corresponding to the insulator capacitance) to a lower capacitance value in depletion at positive bias values. It is noteworthy that at $270 \mathrm{~K}$ the 
two bias sweeps are indistinguishable and even at $330 \mathrm{~K}$ there is only a slight difference between the increasing and decreasing sweep.

An MIS diode can be described as the series capacitance of insulator and depletion layer in the semiconductor. With the maximum capacitance being given by the insulator capacitance $C_{\text {ins }}$ and the minimum total capacitance by the series of $C_{i n s}$ and $C_{s, \min }$ with the minimum capacitance of the depletion layer as

$$
C_{s, \min }=\epsilon_{0} \epsilon_{s} \frac{A}{l_{\max }}
$$

and the depletion length as

$$
l_{d e p}=\sqrt{\frac{\epsilon_{0} \epsilon_{s} \cdot\left|\Psi_{B}\right|}{q N_{A}}} .
$$

Thereby $\epsilon_{s}$ is the relative dielectric constant of the semiconductor, $\epsilon_{0}$ the permittivity of vacuum, $q$ the elementary charge, and $A$ the area of the device. The bulk potential $\Psi_{B}$ is estimated as $0.7 \ldots 0.8 \cdot\left(E_{G} / 2\right)$ with a band gap $E_{G}=2 \mathrm{eV}$ for P3HT. The doping concentration $N_{A}$ can be calculated assuming that the quantity $l_{\max }$ corresponding to the measured capacitance $C_{s, \min }$ is equal to the depletion length $l_{d e p}$. This requires the depletion length $l_{d e p}$ to be smaller the then semiconductor layer thickness $d$. Otherwise one can only obtain an upper limit for $N_{A}$.

Using the standard Schottky-Mott analysis, the doping concentration alternatively can be extracted from the slope of the $C$ - $V$ curves in the transition region between $C_{\max }$ and $C_{\min }$ via 


$$
\frac{\partial}{\partial V}\left(C^{-2}\right)=\frac{2}{\epsilon_{0} \epsilon_{s} q N_{A} A^{2}}
$$

Both analyzes give a constant doping concentration of $10^{16} \mathrm{~cm}^{-3}$ in the analyzed temperature range from 270 to $330 \mathrm{~K}$ in agreement with previous studies by E.J. Meijer et al. [5,8].

Fig. 2(b) shows $C$ - $f$ sweeps with bias in accumulation and depletion at different temperatures. In accumulation one observes a capacitance value corresponding to the insulator capacitance only at low frequency. Depending on the temperature at higher frequency the capacitance shows a so-called relaxation process and drops to the depletion value which is independent of temperature in the investigated range.

The $C$ - $f$ measurements were modeled by the structure shown in the inset of Fig. 2(b) as a series of insulator $\left(R_{\text {ins }}, C_{\text {ins }}\right)$, semiconductor $\left(R_{s}, C_{s}\right)$ and contact or lead resistance $\left(R_{l}\right)$. For this equivalent circuit the bulk relaxation time $\tau_{r}$ is given by

$$
\tau_{r}=R_{s}\left(C_{i n s}+C_{s}\right)
$$

On the one hand, it is required that the frequency for analyzing the $C-V$ curves is much lower than the bulk relaxation frequency $f_{r}=\left(2 \pi \tau_{r}\right)^{-1}$. On the other hand, $f_{r}$ can be used to determine the charge carrier mobility $\mu_{\perp}$ perpendicular to the insulator from the bulk semiconductor resistance $R_{s}$ by

$$
R_{s}=\frac{d}{q N_{A} \mu_{\perp} A}
$$


The resulting mobility of charge carriers varies in the range $1-3 \times 10^{-5} \mathrm{~cm}^{2} / \mathrm{Vs}$ between 270 and $300 \mathrm{~K}$ and is comparable to the mobility obtained from space-charge limited currents in sandwich geometry with two metal electrodes [7]. The hole mobility parallel to the insulator surface was measured in FET structures to be about $4 \times 10^{-4} \mathrm{~cm}^{2} / \mathrm{Vs}$. This difference of mobility between sandwich and in-plane electrode structures is also reported in the literature $[8,9]$ and can be as high as 3 orders of magnitude for P3OT and $\mathrm{OC}_{1} \mathrm{C}_{10}-\mathrm{PPV}$ $[7,9,10]$. While Scheinert et al. ascribe this difference in mobility between the two geometries to a structural and electrical anisotropy of the semiconducting polymer [10], Tanase et al. point out the dependence of the mobility on charge carrier density which is significantly different in FETs and diode structures $[7,9]$, therefore making the anisotropy of the mobility a rather ill-defined quantity. On the other hand structural investigations and charge modulation spectroscopy has indicated that P3HT forms a 2-dimensional electronic system parallel to the gate dielectric [4]. Thus the anisotropy of the charge carrier mobility in FET structures remains an open issue for further investigations.

\subsection{MIS diode with polymeric insulator}

The $C$ - $V$ and $C$ - $f$ characteristics for a Au/P3HT/Polymer insulator/ITO MIS diode is shown in Fig. 3. In contrast to the structures on silicon oxide the capacitance in accumulation and depletion are temperature dependent and both also show a pronounced increase at low frequencies. The main reason for this behaviour is that the capacitance of the polymer insulator alone is already frequency and temperature dependent (however, it does not show a bias dependence). The frequency and temperature dependent dielectric constant $\epsilon_{s}$ 
was determined as 4..5. Thus the analysis following the series of insulator and depletion capacitance is less precise than in the case of $\mathrm{SiO}_{2}$, but as an estimate a doping concentration of about $10^{17} \mathrm{~cm}^{-3}$ is obtained. This is one order of magnitude higher then using silicon oxide insulator indicating that the thermally cross-linked polymeric insulator could cause additional doping of P3HT.

In contrast to the MIS diodes on $\mathrm{Si} / \mathrm{SiO}_{2}$ here a clearly visible temperature dependent hysteresis was observed in $C$ - $V$ curves. This hysteresis is quite small at low temperature but grows rapidly with increasing temperature and is as large as about $10 \mathrm{~V}$ at $330 \mathrm{~K}$. The strong increase is a first hint that mobile ions could be responsible for this behaviour. Further support for this hypothesis comes from a bias-temperature stress experiment as shown in Fig. 4(a). The curves were measured after stressing this device at $350 \mathrm{~K}$ (where ions should be mobile) with different bias voltages $( \pm 40 \mathrm{~V})$ for 60 min and cooling down to $220 \mathrm{~K}$ under applied bias (to freeze in mobile ions). As can be seen there is a large difference between the two curves which can be explained by mobile ions causing a shift of the flat-band voltage depending on the bias stress conditions. Thus both, the temperature dependence of the hysteresis and the possibility to shift the $C-V$ curves after stressing and freezing give strong evidence for the presence of mobile ions. Since this effect is not observed with P3HT on $\mathrm{SiO}_{2}$ we have to conclude that these ions are residing in the polymer insulator. A possible origin could be the initiator necessary for starting the crosslinking process.

The density of mobile ions $Q_{m}[11]$ can be calculated from the shift of the flat-band voltage $\Delta V_{F B}$ between different bias sweep directions as 


$$
Q_{m}=C_{i n s}\left(V_{F B}^{+}-V_{F B}^{-}\right) .
$$

Strictly speaking, the flat-band voltage $V_{F B}^{ \pm}$should be determined at the flatband capacitance which is a series of insulator and Debye capacitance [11]. However, the flat-band capacitance is not used here because there is a huge temperature and frequency dependence of the insulator capacitance. Instead we use as an estimate for $\Delta V_{F B}$ the width of the hysteresis at half value between minimal and maximal capacitance in the $C$ - $V$ curve for each temperature. The resulting amount of charge increases with temperature from less than $10^{10} \mathrm{~cm}^{-2}$ at $280 \mathrm{~K}$ to almost $10^{11} \mathrm{~cm}^{-2}$ at $320 \mathrm{~K}$. In an Arrhenius plot an activation energy for the mobile ions of $0.62 \mathrm{eV}$ can be obtained (see Fig. 4(b)). This large value of the activation energy (varying between about 0.6 and $0.8 \mathrm{eV}$ on different samples) is also indicative for mobile ions as being the origin of hysteresis in MIS diodes using a cross-linked polymeric gate insulator.

\section{Conclusion}

In conclusion we have shown that capacitance measurements on MIS diodes are a powerful tool for the determination of doping concentration, charge carrier mobility and density of mobile ions in polymeric FET structures. The comparison of structures with $\mathrm{SiO}_{2}$ and a thermally cross-linked polymer as gate dielectric indicates that the doping concentration in the organic semiconductor P3HT is one order of magnitude higher with the polymeric insulator than on silicon oxide. Additionally mobile ions in the polymeric gate dielectric are responsible for hysteresis in $C-V$ curves observed for these devices. 


\section{Acknowledgment}

This work was supported by the Deutsche Forschungsgemeinschaft (Focus Programme "Organic Field-Effect Transistors") and the German Bundesministerium für Bildung und Forschung (Focus Programme "Polymer Electronics", POLITAG project). We thank the companies Merck and Philips for providing materials and financial support.

\section{References}

[1] G. Horowitz, Adv. Mat. 10 (1998) 365.

[2] A. R. Brown, C. P. Jarrett, D.M. de Leeuw, and M. Matters, Synth. Met. 88 (1997) 37.

[3] A. Salleo, M. Chabinyc, M.S. Yang, and R.A. Street, Appl. Phys. Lett. 81 (2002) 4383.

[4] H. Sirringhaus, P.J. Brown, R.H. Friend, M.M. Nielsen, K. Bechgaard, B.M.W. Langeveld-Voss, A.J.H. Spiering, R.A.J. Janssen, E.W. Meijer, P. Herwig, and D.M. de Leeuw, Nature 401 (1999) 1038.

[5] E.J. Meijer, A.V.G. Mangnus, C.M. Hart, D.M. de Leeuw, and T.M. Klapwijk, Appl. Phys. Lett. 78 (2001) 3902.

[6] S.M. Sze, Physics of Semiconductor Devices, Wiley, New York, 1982.

[7] C. Tanase, E.J. Meijer, P.W.M. Blom, and D.M. de Leeuw, Phys. Rev. B 91 (2003) 216601.

[8] E.J. Meijer, C. Detcheverry, P.J. Baesjou, E. van Venendaal, D.M. de Leeuw, and T.M. Klapwijk, J. Appl. Phys. 93 (2003) 4831. 
[9] C. Tanase, E.J. Meijer, P.W.M. Blom, and D.M. de Leeuw, Org. Electron. 4 (2003) 33 .

[10] S. Scheinert, and W. Schliefke, Synth. Met. 139 (2003) 501.

[11] B. Streetman, and S. Banerjee, Solid state Electronic Devices, Prentice Hall, 1999. 


\section{Figure Caption}

Figure 1: Schematic structure of an MIS diode (a) with the layer materials and layer thicknesses as well as the structure of regioregular P3HT (b) is shown.

Figure 2: (a) Capacitance-voltage characteristic of an $\mathrm{Au} / \mathrm{P} 3 \mathrm{HT} / \mathrm{SiO}_{2} / \mathrm{Si} \mathrm{MIS}$ diode at $111 \mathrm{~Hz}$ for different temperatures is shown. The arrows indicate the sweep direction of the bias voltage. (b) Capacitance-frequency characteristic of an $\mathrm{Au} / \mathrm{P} 3 \mathrm{HT} / \mathrm{SiO}_{2} / \mathrm{Si} \mathrm{MIS}$ diode in accumulation (temperature dependent) and depletion mode is shown. The arrow indicates the frequency the capacitance-voltage characteristics (Fig. 2(a)) was taken. The inset shows a model structure for simulating resistors and capacitors for contacts, semiconductor and insulator.

Figure 3: (a) Capacitance-voltage characteristic of an Au/P3HT/Polymer insulator/ITO MIS diode at $511 \mathrm{~Hz}$ for different temperatures is shown. The arrows indicate the sweep direction of the bias voltage. (b) Capacitancefrequency characteristic of an Au/P3HT/Polymer insulator/ITO MIS diode for different temperatues in accumulation and depletion mode is shown. The arrow indicates the frequency the capacitance-voltage characteristics (Fig. 3(a)) was taken.

Figure 4: (a) Capacitance-voltage characteristic of the same device like in Fig. 3 was taken at $220 \mathrm{~K}$ after stressing at $\pm 40 \mathrm{~V}$ and $350 \mathrm{~K}$. (b) ArrheniusPlot of charge density determined from the width of hysteresis in Fig. 3(a) for calculation of activation energy of mobile ions is shown. 
(a) $\underline{\mathrm{SiO}}_{2}$ insulator diode

$\mathrm{Au}$

P3HT: $d_{\mathrm{s}} \sim 150 \mathrm{~nm}$

$\mathrm{SiO}_{2}: d_{\text {ins }} \sim 50 \mathrm{~nm}$

$\mathrm{p}^{+}$Si-wafer $(300 \mu \mathrm{m})$

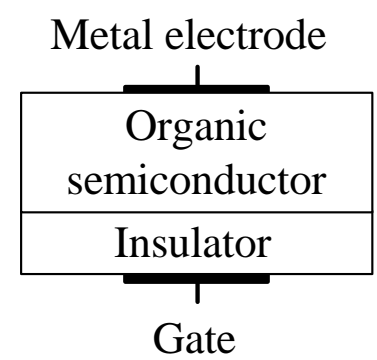

Polymer insulator diode

$\mathrm{Au}$

P3HT: $d_{\mathrm{s}} \sim 150 \mathrm{~nm}$

Polymer insulator: $d_{\text {ins }} \sim 600 \mathrm{~nm}$

ITO on glass

(b)

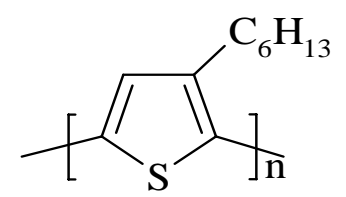

Fig. 1. 


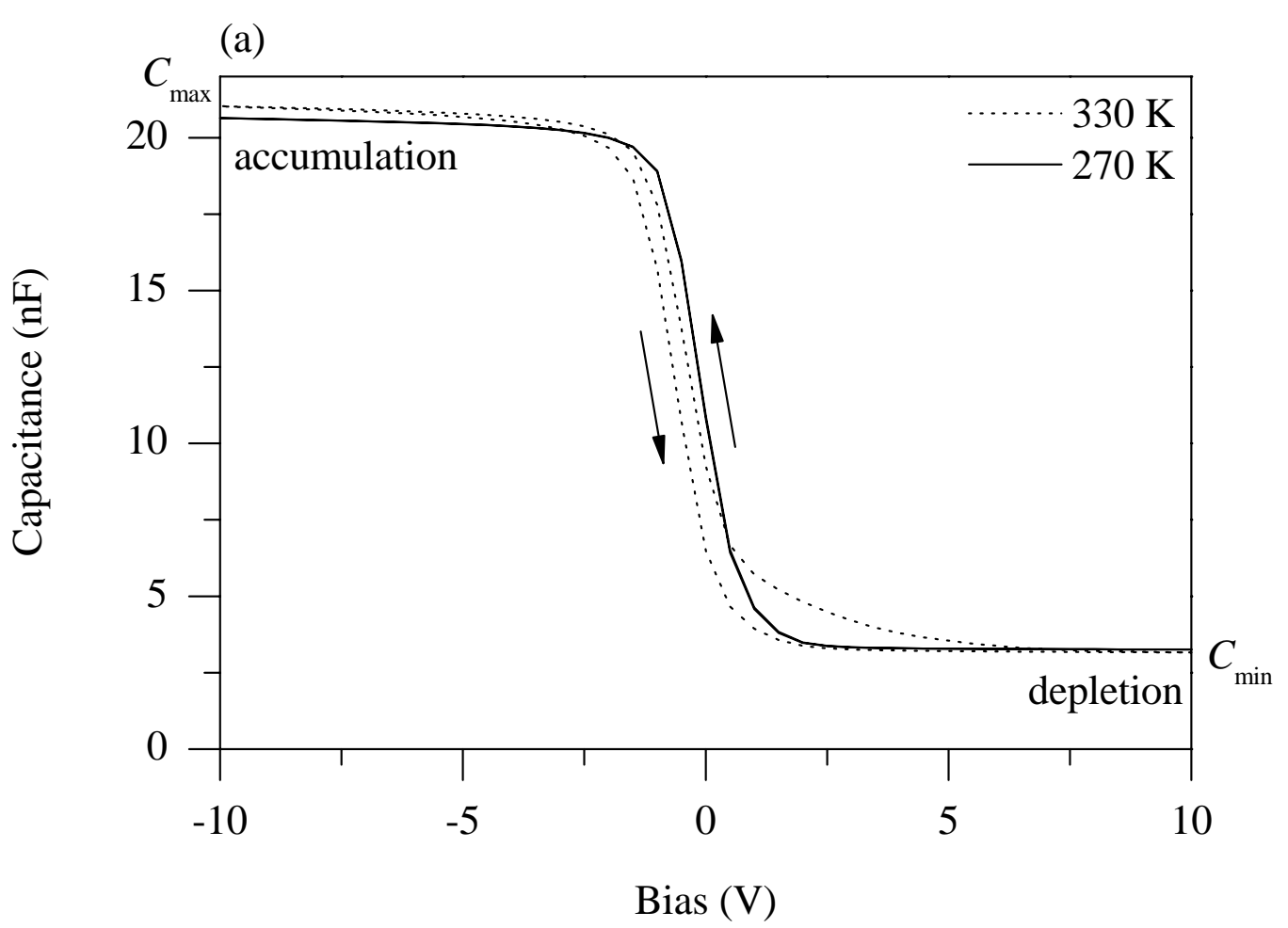

(b)

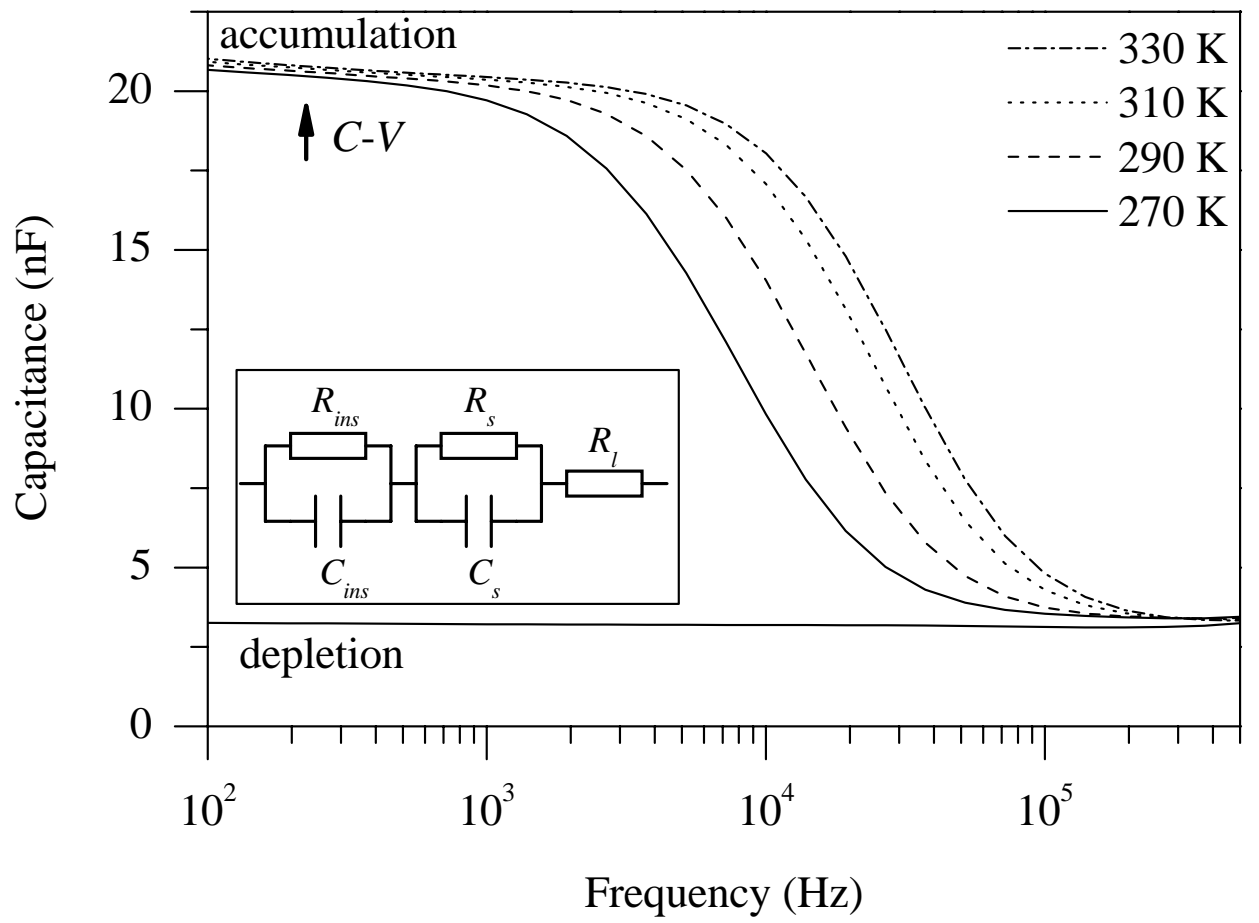

Fig. 2 . 


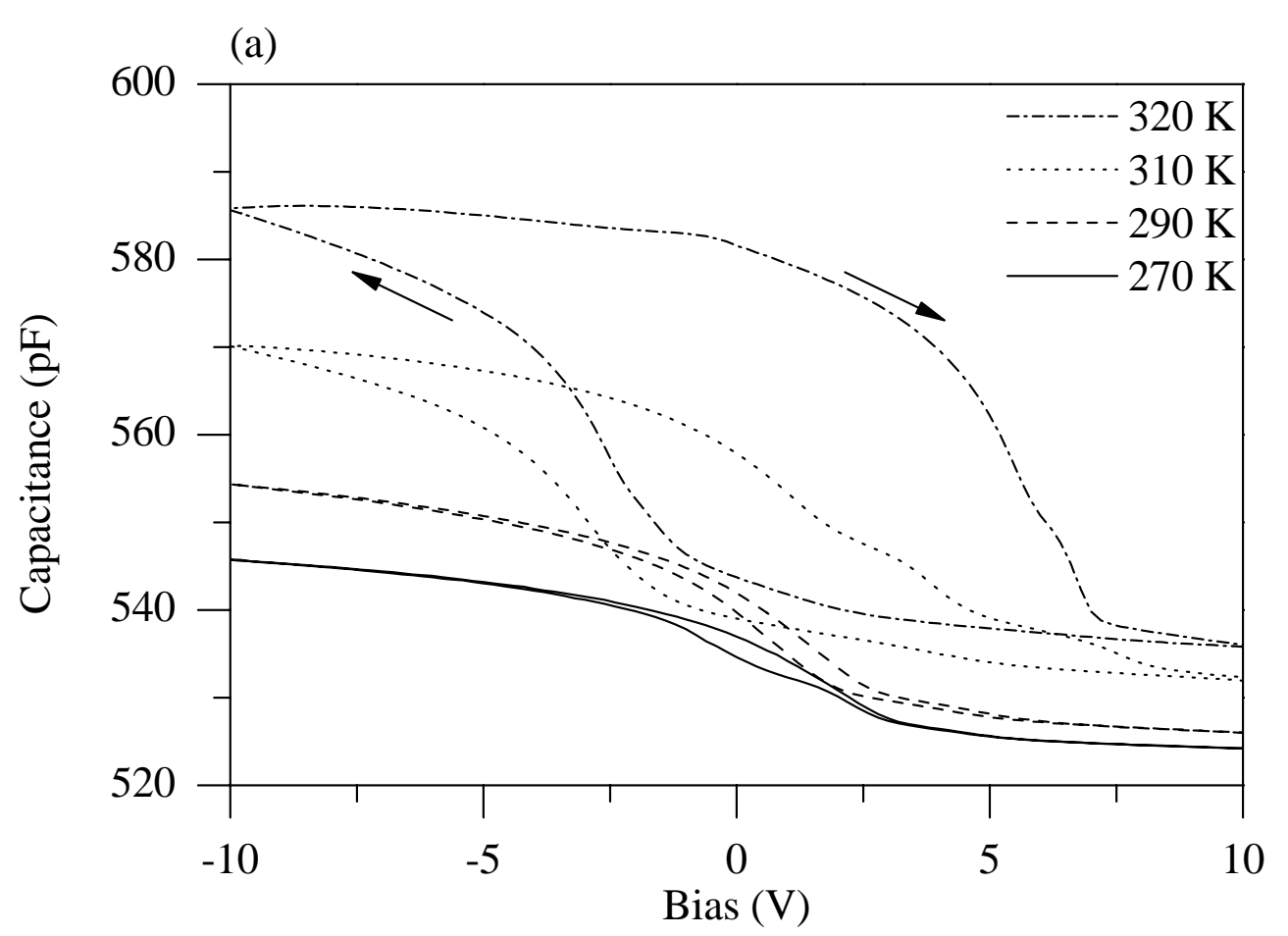

(b)

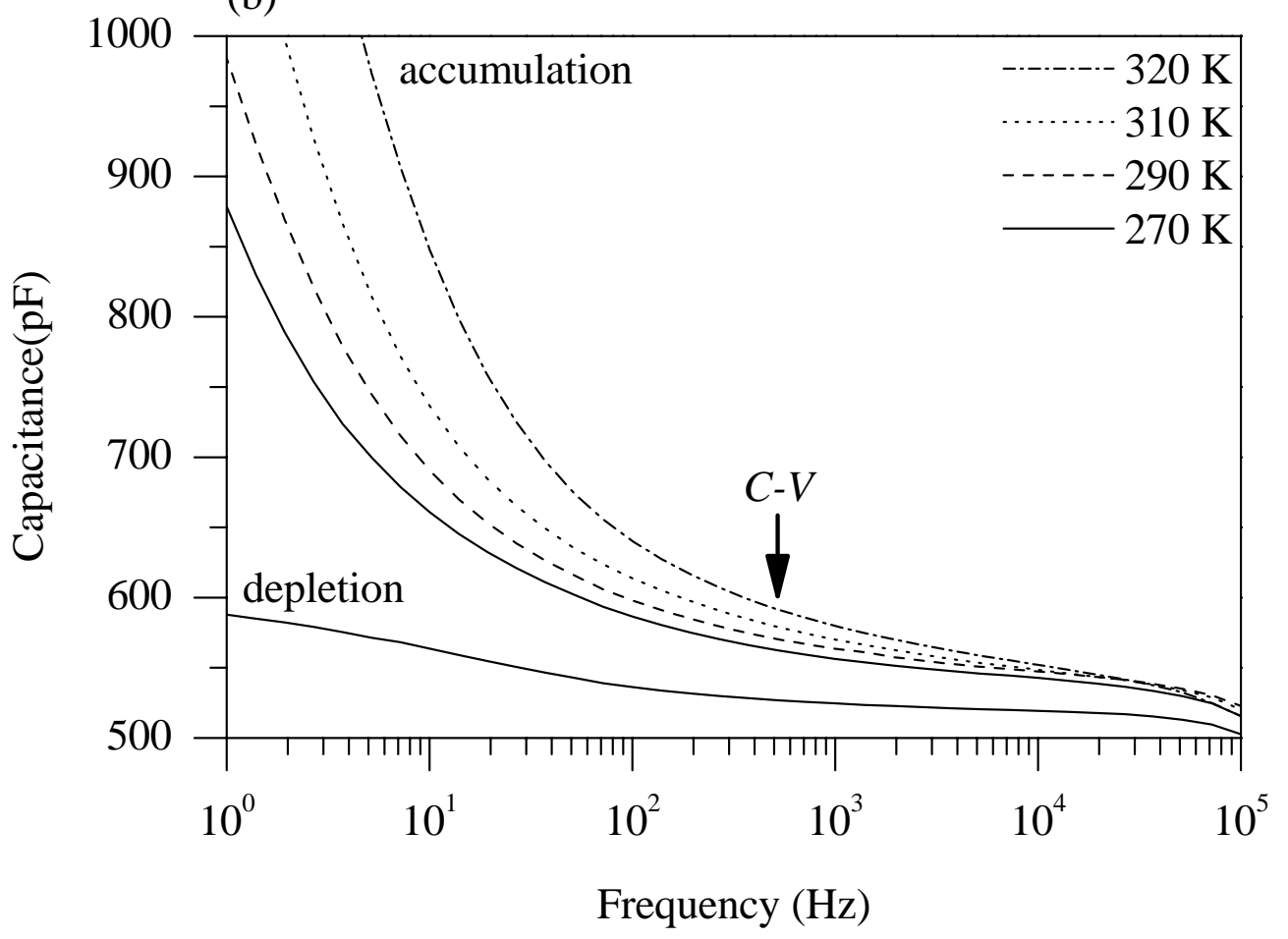

Fig. 3 . 


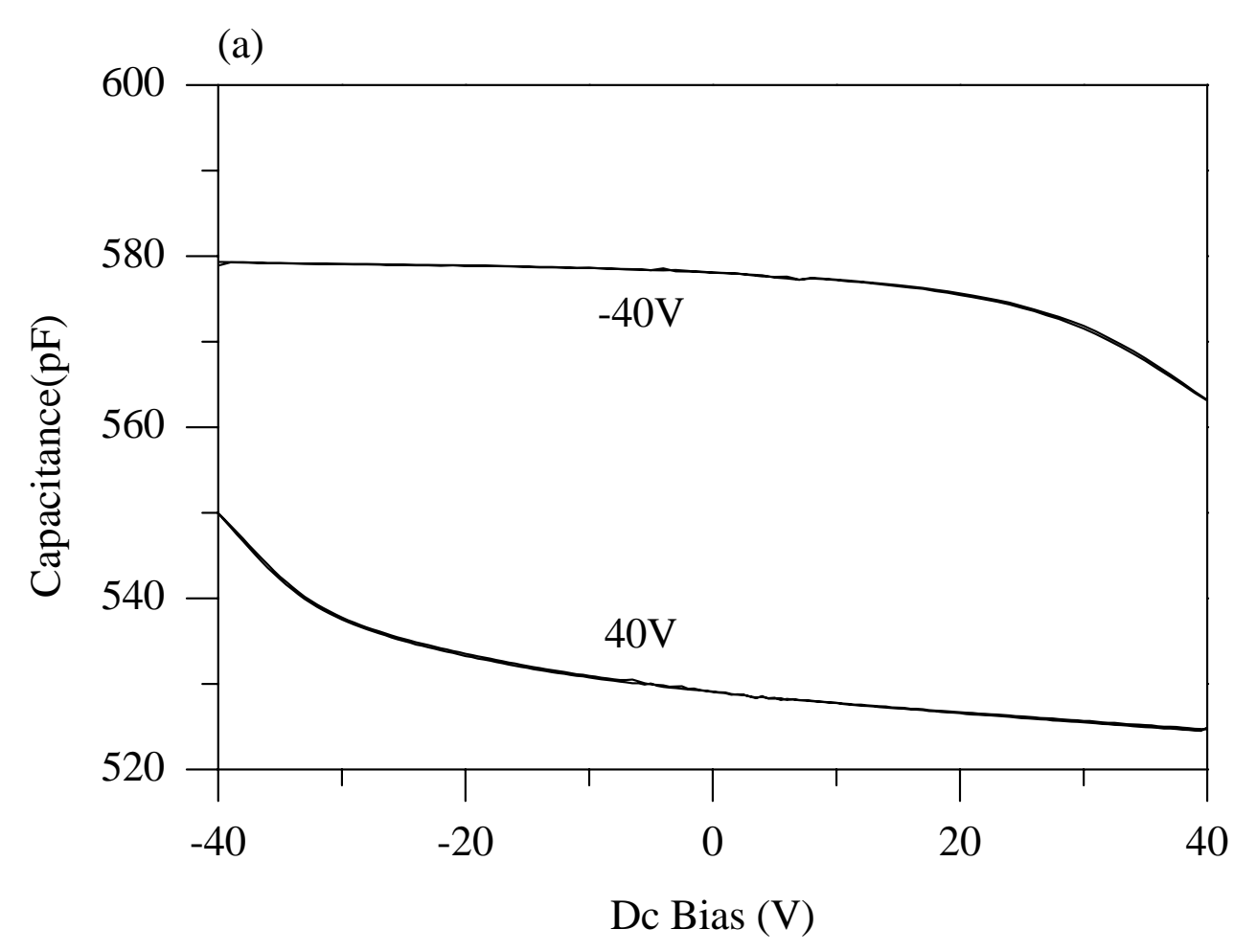

(b)

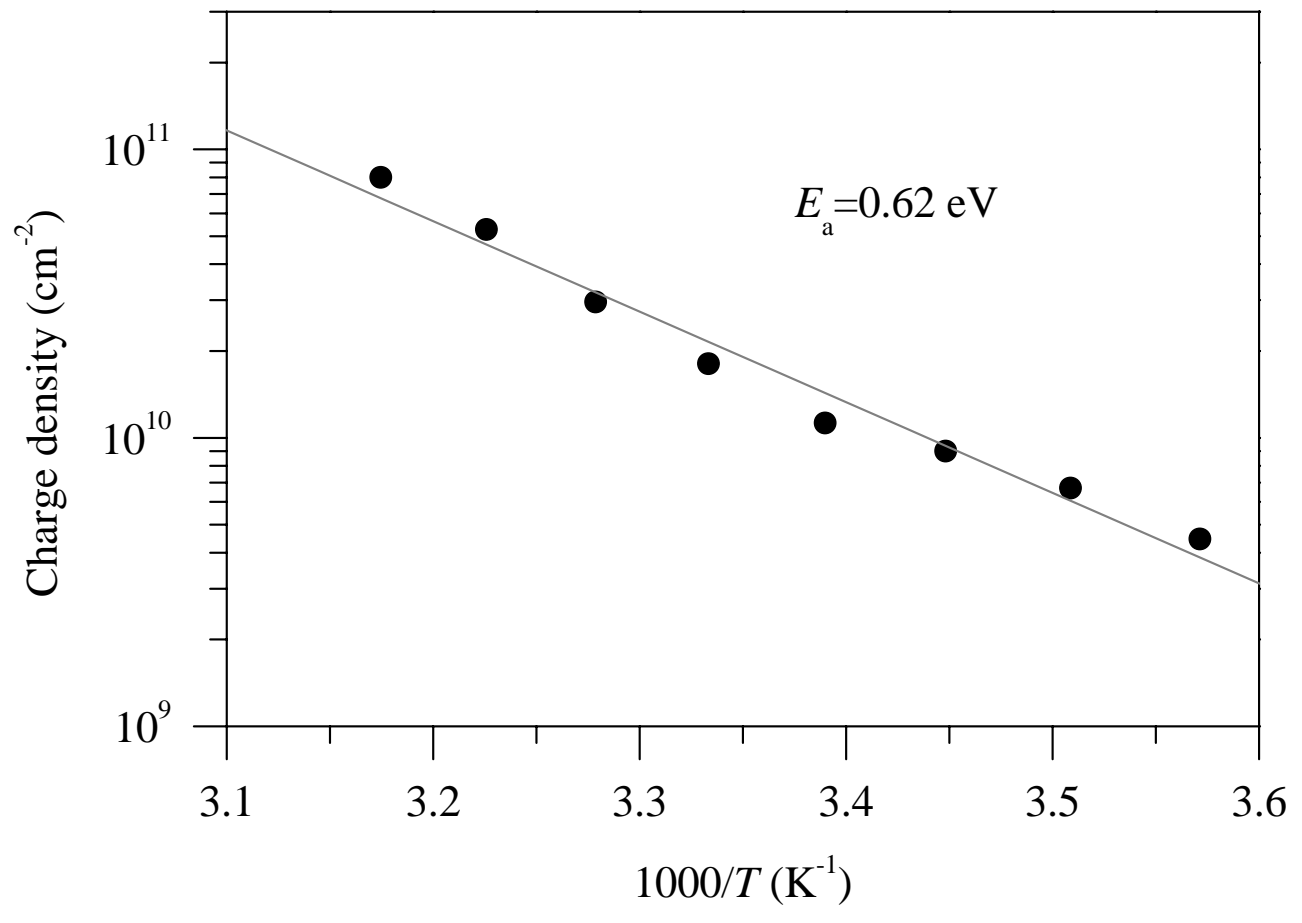

Fig. 4. 